\title{
Atmospheric Moisture Content Effects on Ionic Liquid Wettability of Alumina
}

\author{
S. Brittle, A. Samuel, W. B. Zimmerman \\ Department of Chemical and Biological Engineering, the University of Sheffield.
}

\begin{abstract}
The contact angles or wettability of 7 lonic Liquids, on an alumina substrate, have been measured under two different storage conditions. The first using a small amount of moisture content, the second with no moisture content. The contact angle of lonic Liquid droplets on an alumina substrate were measured using an Attension Theta tensiometer with automated software. The results show that a small amount of moisture improves the wettability of the lonic Liquid - Alumina system and therefore subsequent uses of these liquids with alumina should take this into consideration.
\end{abstract}

\section{Keywords Ionic Liquids; Contact Angle; Wettability; Alumina; Microbubbles}

\section{INTRODUCTION}

An Ionic Liquid (IL) is a liquid that is made up purely of ions and defined as an lonic Liquid if it is liquid below $100^{\circ} \mathrm{C}$. ILS are important solvents for the future and have recently gathered a significant amount of interest from a wide variety of research groups covering wide ranging topics. This interest is due to their flexibility to be engineered into selective solvents with differing features, coupled with their low volatility. Current widely used industrial solvents are volatile organic solvents (VOCs) which are potentially hazardous due to their emission in the atmosphere. Using ILs to replace VOCs would reduce environmental pollution.

The first industrial process to use ILs began in 2003 and since then interest in these "molten salts" has quickly escalated; other industrial applications of ILs have been implemented or are due to be released in the coming years. BASF have reported that their BASIL process was improved by a factor of 80,000 with the use of ILs in their reactors [1].

Other research has focused on the use of ILs as dielectric materials for thin film transistor devices due to their conducting properties, however substrate and nanofabricated thin film degradation has been reported when using ILs [2].

This research intends towards the use of ILs within the chemical separations industry and as a result it is imperative to characterise the behaviour of ILs. This particular study reports the effect of added atmospheric moisture to the contact angle between, or wettability of, ILs and a commonly used micro-porous ceramic diffuser material, alumina.

\section{FOUNDATIONS}

The Young equation which was derived a couple of centuries ago, defines the contact angle $(\theta)$ in a relationship between the surface tensions of the three phases that meet:

$$
\cos \theta=\frac{\gamma_{S V}-\gamma_{S L}}{\gamma_{L V}}
$$

where $\psi$ is the surface tension at the interface of two phases and $S, V$ and $L$ represent the solid, vapour and liquid phases respectively. The Young equation above suggests that contact is independent of drop size, provided that the drop is small enough to ignore gravitational effects [3].
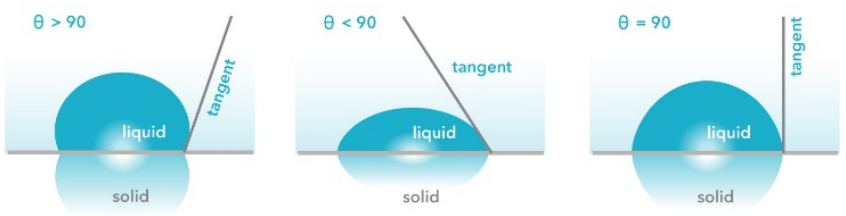

Figure 1. Three different contact angles representing various extents of wettability. (www.attension.com).

Contact angles lower than $90^{\circ}$ indicate that the liquid spreads well across the solid and therefore has high wettability. On the other hand, contact angles greater than $90^{\circ}$ indicates a poor wettability. Both extents of wettability have advantages for different applications.

\section{MethodS}

The 7 ILs were kept in two different environments. In the dry environment the ILs were heated to $60^{\circ}$ for 24 hours in order to remove residual moisture and then these ILs were kept under a vacuum in a desiccator with drying salts in order to maintain the state of removed moisture. Under wet conditions the 7 ILs were kept within regular laboratory conditions where they are exposed to atmospheric moisture for long periods of time.

An Attension Theta tensiometer was used to measure the contact angle of 7 ILs. These ILs were randomly chosen to represent a broad range of available ILs. The equipment is able to take precise measurements of the contact angle using an optimised setup including a monochromatic light source, adjustable sample; and sophisticated software able to recognise the drop and measure its contact angle automatically. The ILs were pipetted onto the cleaned alumina substrate stage, adjustable in 3 dimensions. The droplet is then centred and an image taken for analysis by the Theta droplet software. An averaged contact angle is calculated from the recorded imaging taking the left and right angles into consideration (within $5 \%$ of each other or symmetry).

The alumina substrates were cleaned a using an ultrasonic bath and a series of three solvent (chloroform, isopropyl- 
alcohol and water) and repeated in reverse order to ensure a clean surface to measure IL contact angles.

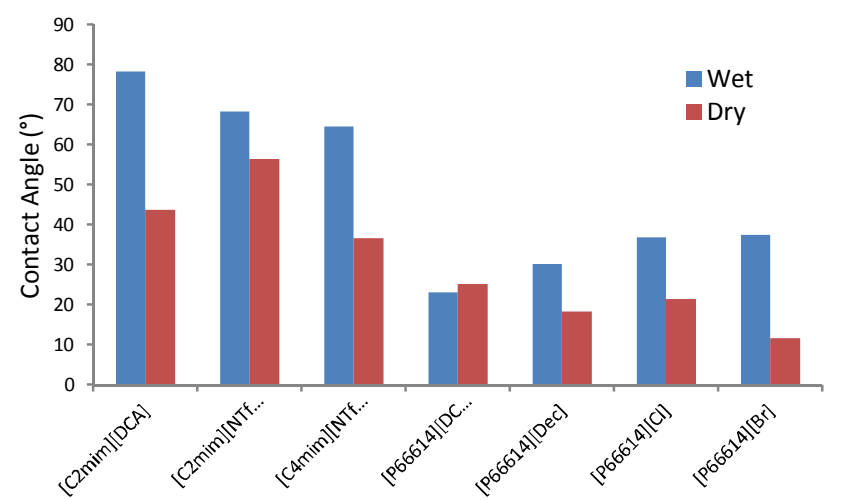

Figure 2. The contact angles of 7 ILs on an alumina substrate, when ILs are stored under wet (blue) and dry (red) conditions.

Fig. 2 shows how the contact angle of the 7 ILs varies with different storage conditions, allowing for varying volumes of moisture content. The overall result indicates that ILs with added water content improve the wettability on an alumina substrate. This result can be important because it indicates the optimum conditions for increased wettability is with lower water content.

Table 1. Contact angle and viscosity data for the 7 ILs.

\begin{tabular}{|c|c|c|c|}
\hline Ionic Liquid & $\begin{array}{l}\text { Wet } \\
\text { Contact } \\
\text { Angle }\left({ }^{\circ}\right)\end{array}$ & $\begin{array}{l}\text { Dry } \\
\text { Contact } \\
\text { Angle }\left({ }^{\circ}\right)\end{array}$ & $\begin{array}{l}\text { Viscosity } \\
\text { (Pa-s) }\end{array}$ \\
\hline$\left[C_{2} \operatorname{mim}\right][D C A]$ & 78.3 & 43.7 & 0.03 \\
\hline$\left[\mathrm{C}_{2} \mathrm{mim}\right]\left[\mathrm{NTf}_{2}\right]$ & 68.4 & 56.4 & 0.0386 \\
\hline$\left[\mathrm{C}_{4} \mathrm{mim}\right]\left[\mathrm{NTf}_{2}\right]$ & 64.5 & 36.7 & 0.0515 \\
\hline$\left[\mathrm{P}_{66614}\right][\mathrm{DCA}]$ & 23.1 & 25.2 & 0.2804 \\
\hline$\left[\mathrm{P}_{66614}\right][\mathrm{Dec}]$ & 30.3 & 18.5 & 0.3189 \\
\hline$\left[\mathrm{P}_{66614}\right][\mathrm{Cl}]$ & 37 & 21.6 & 1.824 \\
\hline$\left[\mathrm{P}_{66614}\right][\mathrm{Br}]$ & 37.6 & 11.7 & 2.094 \\
\hline
\end{tabular}

Table 1 shows the wet and dry contact angle data. The larger contact angles and thus improved wettability are found in the lower viscosity ILs ( $<0.06 \mathrm{~Pa}-\mathrm{s})$, however this trend does not extend to the higher viscosity ILs (>1.00 Pa-s) since there is somewhat of an overlap with the midrange viscosity ILs (0.06 Pa-s to $1.00 \mathrm{~Pa}-\mathrm{s}$ ). This suggests that although the viscosity may suggest the extent of wettability there are also other parameters such as surface tension, that may be influencing the contact angle. This should be concern for further investigation, in order to determine the role of various parameters of IL such as the Kamlet-Taft solvent descriptors (alpha, beta and $\mathrm{pi}^{*}$ ).

\section{Conclusions}

Wet ILs have been found to improve wettability of ILs on an alumina surface. This must be considered when using ILs and microbubbles in separation processes, since microbubble sizes can be reduced with increased wettability. Further investigation in the parameters which control wettability must be undertaken in order to produce IL with optimum characteristics for their application.

\section{ACKNOWLEDGEMENTS}

The authors would like to acknowledge the 4CU program and the EPSRC for the funding of this work.

\section{REFERENCES}

1. Rodgers R, Seddon K. Ionic Liquids - Solvents of the Future? Science. 2003. pp792-793.

2. Chen F, Qing Q, Xia J, Li J, Tao N., Electrochemical GateControlled Charge Transport in Graphene in lonic Liquids, J. Am. Chemical Society, 2009; 131: p29.

3. Quere D. Rough Ideas on Wetting. Physica A. 2002; 313: p. 32$\underline{46 .}$ 